\title{
Formation of soliton complexes in dispersive systems
}

\author{
M.M.Bogdan ${ }^{1}$, A.M.Kosevich ${ }^{1}$, G.A.Maugin ${ }^{2}$ \\ 1 B.I.Verkin Institute for Low Temperature Physics and Engineering \\ of the National Academy of Sciences of Ukraine, \\ 47 Lenin Ave., 310164, Kharkov, Ukraine \\ 2 Laboratoire de Modélisation en Mécanique, Université Pierre et Marie \\ Curie, Boite 162, Tour 66, 4 Place Jussieu, 75252 Paris Cedex 05, France
}

Received September 15, 1998

The concept of soliton complex in a nonlinear dispersive medium is formulated. It is shown that interacting identical topological solitons in the medium can form bound soliton complexes which move without radiation. This phenomenon is considered to be universal and applicable to various physical systems. The soliton complex and its "excited" states are described analytically and numerically as solutions of nonlinear dispersive equations with the fourth and higher order spatial or mixed derivatives. The dispersive sine-Gordon, double and triple sine-Gordon, and piecewise models are studied in detail. Mechanisms and conditions of the formation of soliton complexes, and peculiarities of their stationary dynamics are investigated. A phenomenological approach to the description of the complexes and the classification of all the possible complex states are proposed. Some examples of physical systems, where the phenomenon can be experimentally observed, are briefly discussed.

Key words: soliton complex, dispersive medium, radiationless motion

PACS: $47.35 .+i, 02.70 .-c, 47.20 . K y, 62.30 .+d, 03.40 . K f$

\section{Introduction}

Discrete and continuum models of the condensed matter exhibit universal nonlinear effects which can manifest themselves in dispersive media such as microstructured solids, macroscopic discrete systems (arrays of Josephson junctions, nonlinear transmission lines and so on), fluids and dissipative-dispersive systems [1-6].

One of these effects, occurring in the dynamics of nonlinear excitations of a dispersive medium, is the formation of bound states of solitons. This phenomenon of soliton bunching was observed through numerical simulations beginning with the work [4], and studied extensively during last decade [6-12]. 
The role of dispersion as a factor influencing the interaction between well-separated non-topological solitons was discussed in works [7,8]. Interactions of oscillating soliton tails in dispersive media lead to the formation of bunches of solitons, consisting of two or more well-distinguishable humps. In these theories the contribution of the dispersion to the soliton interaction can be considered as a weak perturbation.

In the present paper we concentrate just on the case of strong interaction between topological solitons. This occurs when identical solitons are closely placed. As a result the repulsive potential of the solitons grows rapidly with decreasing the distance between them. At the same time one has to take into account the dispersion, as an additional source of strong interaction, and this can change the character of the interaction between solitons in general.

The aim of this paper is to show that solitons in a strongly dispersive medium possess an internal structure and their interaction depends on intrinsic properties such as flexibility. Due to this dependence the potential energy turns out to be a nonmonotonic function of the distance between solitons. As a result identical solitons can attract each other and form a bound-soliton complex [9]. Such a complex can move without any radiation in strongly dispersive media $[9,10]$.

We present a number of dispersive models bearing such soliton complexes. The models are described by nonlinear equations with fourth and higher spatial or mixed derivatives. Solutions of the relevant equations can be obtained numerically and analytically. We found exact analytical solutions for two variants of the dispersive sine-Gordon (dSG) and double sine-Gordon equations (dDSG). We also show that the complex, consisting of three solitons, can be described explicitly in the dispersive equations with the additional sixth derivative in the cases of the sine-Gordon and triple sine-Gordon models.

Topological soliton complexes can also be realized as discrete sets of solitonic configurations with internal structures. We propose the classification of these "excited" states of soliton complexes, constructing them explicitly in the framework of the double piecewise dispersive model. The two-soliton ansatz approximation is used to establish analytically the existence condition for the soliton complex in the dSG and dDSG equations. We find numerical solutions of these equations for the two-soliton complex and its "excited" states, and their dependencies of energies and velocities on the model parameters. As a result we formulate the concept of the soliton complex and classify it as a specific bound state of identical solitons in a strongly dispersive medium.

\section{Dispersive models and soliton-complex solutions}

A large variety of physical processes in solids is described by the discrete sineGordon equation. We write it in the dimensionless form following the notations of the work [1]:

$$
\frac{\partial^{2} u_{n}}{\partial \tau^{2}}+2 u_{n}-u_{n-1}-u_{n+1}+\frac{1}{d^{2}} \sin \left(u_{n}\right)=0,
$$


where $u_{n}$ is, e.g., the displacement of atom $n$ and $d$ is the discreteness parameter. In this highly discrete system Peyrard and Kruskal observed by means of a numerical simulation the almost radiationless motion of the $4 \pi$-soliton. At the same time stationary motion of a single $2 \pi$-soliton was impossible in this system because of a strong radiation emitted by the moving $2 \pi$-soliton [2].

In the work [9] it was found that the radiationless motion of the complex can be described explicitly in the framework of the dispersive sine-Gordon equation (1dSG) with a fourth spatial derivative:

$$
u_{t t}-u_{x x}-\beta u_{x x x x}+\sin (u)=0 .
$$

Equation (2) is obtained as the long wave limit of equation (1) by substituting $x d$ for $n, t d$ for $\tau$, and the second difference by the series:

$$
u_{n-1}+u_{n+1}-2 u_{n} \approx u_{x x}+\beta u_{x x x x}+\ldots .
$$

The relation between the dispersive factor $\beta$ and the discreteness parameter $d$ is $\beta \equiv 1 /\left(12 d^{2}\right)$. The exact solution of equation (2), corresponding to the soliton complex, has the following form:

$$
\begin{gathered}
u_{4 \pi}=8 \arctan \left\{\operatorname { e x p } \left(\sqrt{\left.\left.\frac{2}{3} \frac{x-V_{0} t}{\sqrt{1-V_{0}^{2}}}\right)\right\},}\right.\right. \\
V_{0}= \pm \sqrt{1-\sqrt{\frac{4 \beta}{3}}} .
\end{gathered}
$$

The velocity of the complex is not an arbitrary constant in the solution (4), but it is a function of the dispersive parameter $\beta$. As a function of the discreteness parameter, it equals $V_{0}(d)= \pm \sqrt{1-(1 / 3 d)}$ and differs less than five per cent from numerical result of Peyrard and Kruskal.

It is evident [10] that similar solutions are available for the second or "regularized" dispersive sine-Gordon equation (2dSG) which has the fourth spatio-temporal mixed derivative instead of the fourth spatial derivative of equation (2):

$$
u_{t t}-u_{x x}-\beta u_{t t x x}+\sin (u)=0 .
$$

The form of the soliton complex solution of the equation is the same as for the dSG equation, however the velocity dependence on the parameter $\beta$ differs from equation $(5)$.

$$
V_{r}(\beta)= \pm\left(\sqrt{1+\frac{\beta}{3}}-\sqrt{\frac{\beta}{3}}\right) .
$$

The presence of freely moving soliton-complex structures was also examined numerically in the continuous nonlocal sine-Gordon model [6] described by:

$$
u_{t t}+\sin (u)=\frac{\partial}{\partial x} \int_{-\infty}^{\infty} \mathrm{d} x^{\prime} G\left(x-x^{\prime}\right) u_{x}^{\prime}\left(x^{\prime}, t\right) .
$$


The soliton complex with the internal structure was practically found by Peyrard et al [12] in simulations of the modified sine-Gordon model.

Now we show that soliton complexes exist in the dispersive double sine-Gordon equations. These equations describe a large variety of physical systems: ferro- and antiferromagnets, magneto-elastic systems, superfluid ${ }^{3} \mathrm{He}$ and others [13]. Besides they contain dispersive sine-Gordon equations as the limit cases. Thus we deal with the dispersive double sine-Gordon equation (1dDSG):

$$
u_{t t}-u_{x x}-\beta u_{x x x x}+\sin (u)+2 h \sin (u / 2)=0,
$$

and its regularized variant $(2 \mathrm{dDSG})$ :

$$
u_{t t}-u_{x x}-\beta u_{t t x x}+\sin (u)+2 h \sin (u / 2)=0,
$$

where, for example, in magnetic applications $\phi(x, t)=\frac{1}{2} u(x, t)$ denotes the azimuth angle of the magnetization vector in the easy-plane ferromagnet, and $h$ is a magnetic field applied along the easy plane. When $h=0$ the equations revert to the dispersive and regularized sine-Gordon equations.

Equation (9) can be derived from the Lagrangian:

$$
L=\int_{-\infty}^{\infty} \frac{1}{2}\left\{u_{t}^{2}-u_{x}^{2}+\beta u_{x x}^{2}-2(1-\cos (u))-8 h(1-\cos (u / 2))\right\} \mathrm{d} x .
$$

The Hamiltonian of the dispersive double sine-Gordon system is given by:

$$
H=\int_{-\infty}^{\infty} \frac{1}{2}\left\{u_{t}^{2}+u_{x}^{2}-\beta u_{x x}^{2}+2(1-\cos (u))+8 h(1-\cos (u / 2))\right\} \mathrm{d} x .
$$

Corresponding expressions for the regularized equation (10) are obtained by substituting $u_{t x}^{2}$ for $u_{x x}^{2}$ in equations (11) and (12).

The difference between the dDSG equations results in two types of spectra of linear excitations and different stability properties. The regularized equation is introduced in order to avoid the instability with respect to short-wave perturbations.

We present exact solutions for the dispersive double sine-Gordon equations and discuss the peculiarities of their dynamics. For the concrete definition we imply the magnetic applications of the equations, where solitons are domain walls and parameter $h$ is a magnetic field. Therefore we use this terminology hereinafter.

It is known that applying a magnetic field to a ferromagnet leads to coupling $180^{\circ}$ domain walls into $360^{\circ}$ domain wall. In terms of the usual double sine-Gordon equation $(\beta=0)$ this means the existence of a wobbler solution [13] or the $4 \pi$ kink. Such a static solution does not exist for equation (9) if $\beta \neq 0$ but it holds for equation (10):

$$
u_{w}(x)=4 \arctan \left\{\exp \left(q_{w} x-R_{w}\right)\right\}+4 \arctan \left\{\exp \left(q_{w} x+R_{w}\right)\right\},
$$

where $q_{w}=\sqrt{(1+h)}$ and $\sinh \left(R_{w}\right)=1 / \sqrt{h}$. 
Thus both factors, dispersion and magnetic field, cause the soliton coupling, and it is interesting to investigate their mutual influence on the binding process.

In this work we are interested in a stationary soliton motion, i.e. consider the solutions of the form $u(x, t)=u(x-V t)$. Then both the equations (9) and (10) are reduced to the ordinary differential equation:

$$
u_{z z}+\alpha u_{z z z z}-\sin (u)-2 h \sin (u / 2)=0 .
$$

Here $z=(x-V t) / \sqrt{1-V^{2}}$, and parameter $\alpha$ equals to $\alpha^{(1)}\left(\alpha^{(2)}\right)$ for the $1 \mathrm{dDSG}$ (2dDSG) equation, where

$$
\alpha^{(1)}=\frac{\beta}{\left(1-V^{2}\right)^{2}}, \quad \alpha^{(2)}=\frac{\beta V^{2}}{\left(1-V^{2}\right)^{2}} .
$$

When $h=0$, equation (14) is reduced to the dSG case which was analysed in $[9,10]$.

When $h \neq 0$ we are able to find again the exact solution of equation (14):

$$
u_{4 \pi}=8 \arctan \left\{\exp \left(\sqrt{\frac{2}{3}+h} \frac{x-V t}{\sqrt{1-V^{2}}}\right)\right\} .
$$

Thus the 1dDSG and 2dDSG equations have complex solutions in identical form but with different expressions for the velocities

$$
\begin{gathered}
V_{1}(\beta, h)= \pm \sqrt{1-(2+3 h) \sqrt{\frac{\beta}{3}}} \\
V_{2}(\beta, h)= \pm\left(\sqrt{1+\frac{\beta}{3}\left(1+\frac{3}{2} h\right)^{2}}-\sqrt{\frac{\beta}{3}}\left(1+\frac{3}{2} h\right)\right) .
\end{gathered}
$$

These solutions describe complexes consisting of two strongly bound $2 \pi$-solitons. They differ from the wobbler equation (13) by the effective widths, the zero distance between solitons, and the ability to move in the dispersive medium. Since now velocities are functions of parameter $h$, we can change them from the maximum values corresponding to the dSG limit (equation (5) and equation (7)) to zero. In equation (9) it occurs at the finite critical value $h_{\mathrm{cr}}=\sqrt{\frac{1}{3 \beta}}-\frac{2}{3}$. For example, in ferromagnets we can control the velocity of a motion of the domain wall complex through the magnetic field.

The next peculiarity of the complex dynamics is revealed, if we evaluate the energies using the Hamiltonian expressions (see equation (12)). For the 1dDSG complex we obtain:

$$
E_{1}=32\left[(3 \beta)^{-\frac{1}{4}}-\frac{2}{9}(3 \beta)^{\frac{1}{4}}\right] .
$$

The energy of the soliton complex turns out to be independent of the parameter $h$, and, therefore, of the velocity at the fixed $\beta$ ! This means that, at least, by adiabatic variation of the magnetic field, we can vary the form and speed of the complex, conserving its energy. This remarkable property could be used in the energy transfer 
applications in physical systems described by the 1dDSG equation. However, the property is very much sensitive to spectrum characteristics of the dispersive medium. The regularized equation (12) gives the velocity and field dependent energy:

$$
E_{2}=32\left[\left(3 \beta V_{2}^{2}\right)^{-\frac{1}{4}}-\frac{2}{9}\left(3 \beta V_{2}^{2}\right)^{\frac{1}{4}}\right]
$$

where $V_{2}(\beta, h)$ is given by the expression (18).

Some important properties of the dSG and dDSG equations can be reproduced by the following model equation which we call the dispersive double piecewise equation:

$$
u_{t t}-u_{x x}-\beta u_{x x x x}+f(u)=0,
$$

where force $f(u)$ is a periodic function of the period $4 \pi$. On the interval $[-2 \pi, 2 \pi]$ it is given by

$$
f(u)= \begin{cases}(1+h)(u+2 \pi), & -2 \pi<u<-u_{0} \\ (1-h) u, & |u|<u_{0} \\ (1+h)(u-2 \pi), & u_{0}<u<2 \pi\end{cases}
$$

The point, corresponding to the energy maximum, is chosen as $u_{0}=2 \arccos (h)$ to model the behaviour of the double sine-Gordon model. As $h=0$ and $h=1$ the equation degenerates into the analogue of the dSG equation with the periods $2 \pi$ and $4 \pi$, respectively. This model enables us to construct explicitly the expressions for the soliton complexes and their "excited" states.

As in the dDSG case, after introducing the coordinate $z$ in the moving reference frame, we derive the equation:

$$
u_{z z}+\alpha u_{z z z z}-f(u)=0
$$

where $f(u)$ is given by the expression equation (22). We are interested in odd solutions of equation (23), $u(-z)=-u(z)$, with limit conditions $u( \pm \infty)= \pm 2 \pi$. To construct the $4 \pi$-soliton it is sufficient to find the solution in the external region $\left(z>z_{0}\right)$ and the general odd solution in the internal region $\left(|z|<u_{0}\right)$. They look as follows:

$$
u_{e}(z)=2 \pi-A \exp \left(-\kappa_{2} z\right)
$$

and

$$
u_{i}(z)=B \sin \left(k_{1} z\right)+C \sinh \left(\kappa_{1} z\right) .
$$

Here, for the sake of simplicity we present principal results in the limit case $h=0$. Then the exponent $\kappa_{2}$ coincides with the parameter $\kappa_{1}$ of the solution (25):

$$
\kappa_{1}=\kappa_{2}=\kappa= \pm \sqrt{\left[(1 / 4+\alpha)^{1 / 2}-1 / 2\right] / \alpha} .
$$

The parameter $k_{1}$ equals to:

$$
k_{1}=k=\sqrt{\left[(1 / 4+\alpha)^{1 / 2}+1 / 2\right] / \alpha}
$$




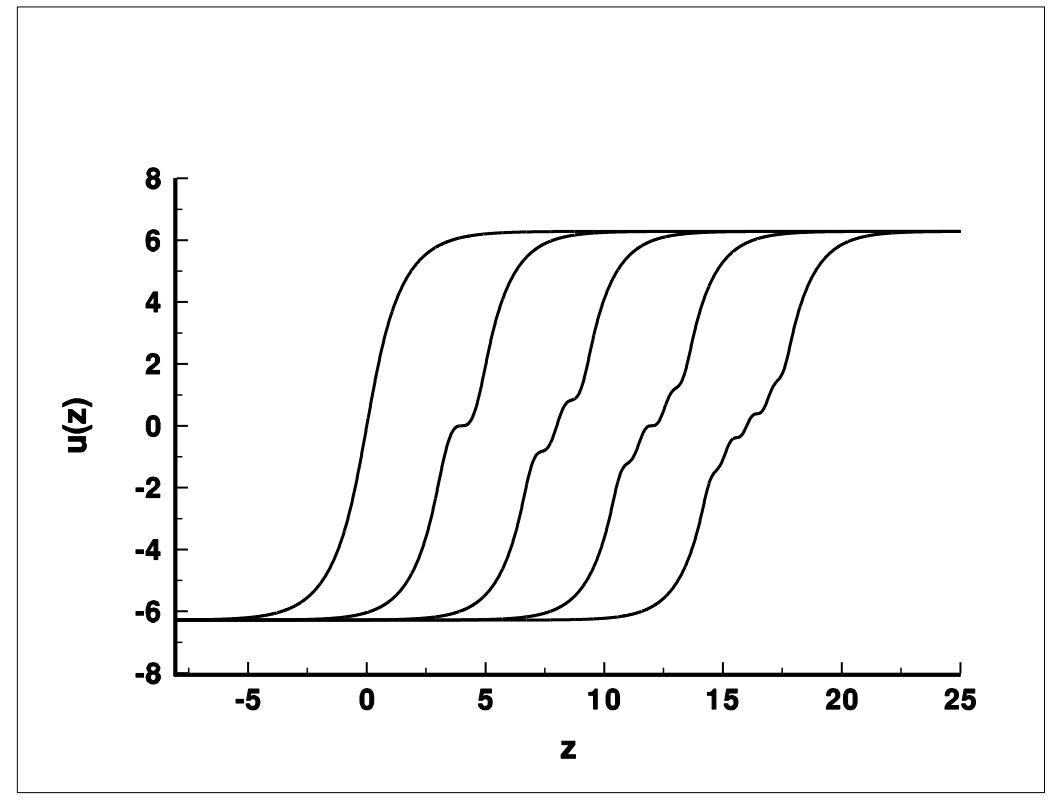

Figure 1. Soliton complex and its "excited" states in the piecewise model.

Using the conditions of continuity of the function $u$ and its first, second and third derivatives in the point $z_{0}$, where $u=u_{0}$, we finally obtain the following equation

$$
\lambda=\exp \left(-\lambda \mu_{n}\right)
$$

where $\lambda(\alpha)=\kappa(\alpha) / k(\alpha)$ and $\mu_{n}=\pi(n-1 / 2)$. This equation determines the eigenvalues $\lambda_{n}$ and $\alpha_{n}$ through the Lampert's function, and makes it possible to find corresponding values of coefficients $A, B$ and $C$, and to construct explicit forms of the complex solution and their "excited" states.

Evidently, the soliton complex states can be classified by the integer values of the parameter $n$. In the "excited" complexes the value of $n$ shows the number of nodes of the oscillating part of the solution. The latter corresponds to the radiation locked between two composite solitons. The first five eigenfunctions are shown below in the figure, where they are numbered from the left to the right by $n=1,2,3,4,5$.

Thus the simple piecewise model exhibits main peculiarities of soliton complex structure and its stationary dynamics, which turn out to be universal for the dispersive nonlinear models.

The next natural question is the existence of an exact solution for soliton complexes consisting of more than two solitons. Numerical integrations of the discrete sine-Gordon equation equation (1) and the non-local model equation (8) reveal such solutions. We are able to find the analytical solution describing the three-soliton complex in the dispersive sine-Gordon equation with sixth spatial derivative:

$$
u_{t t}-u_{x x}-\beta u_{x x x x}-\gamma u_{x x x x x x}+\sin (u)=0 .
$$

The equation can be derived from a discrete model if we take into account the higher-order term in an expansion of the type of equation (3). For the special choice 
of the parameters $\gamma=\frac{3}{20} \beta^{\frac{3}{2}}$, equation (29) has the following exact solution:

$$
u_{6 \pi}=12 \arctan \left\{\exp \left(\sqrt{\frac{23}{45}} \frac{x-V_{*} t}{\sqrt{1-V_{*}^{2}}}\right)\right\},
$$

where the velocity takes the form:

$$
V_{*}= \pm \sqrt{1-\frac{23}{30} \sqrt{\beta}} .
$$

The exact solution of the form of equation (30) also exists in the dispersive triple sine-Gordon equation:

$$
u_{t t}-u_{x x}-\beta u_{x x x x}-\gamma u_{x x x x x x}+\sin (u)+h_{1} \sin \left(\frac{u}{3}\right)+h_{2} \sin \left(\frac{2}{3} u\right)=0,
$$

which is the generalization of equation (29), and where parameters $h_{1}$ and $h_{2}$ are arbitrary constants. Under the condition $\gamma=\frac{3}{20} \beta^{\frac{3}{2}}\left(1+\frac{1}{2} h_{2}\right)$ the three-soliton complex is described by the expression:

$$
u_{6 \pi}=12 \arctan \left\{\exp \left(q_{3}\left(x-V_{3} t\right)\right\} .\right.
$$

The relations between solution parameters are the following:

$$
\begin{gathered}
q_{3}=\left(\frac{4+2 h_{2}}{9 \beta}\right)^{1 / 4} \\
V_{3}= \pm \sqrt{1-\left(\frac{23}{15}+h_{1}-\frac{8}{3} h_{2}\right) \sqrt{\frac{\beta}{4+2 h_{2}}}} .
\end{gathered}
$$

In previous formulas for the presentation of exact solutions we have used the Lorentzinvariant-like expressions to keep the analogy with those of the Lorentz-invariant equations with $\beta=0$. However the solutions can also be written in the form similar to equation (33) which is evidently simpler.

Other dispersive equations combining the properties of the Klein-Gordon and Boussinesq-type equations are known $[11,14]$, in which topological solitons are shown analytically and numerically to possess an internal structure, and, therefore, they are good candidates for bearing soliton complexes.

All the above facts collected together prove that the formation of the bound soliton complex is a universal property of the strongly dispersive media.

\section{Phenomenology and numerical analysis of soliton complex}

We proposed in $[9,10]$ an analytical approach to the description of the solitoncomplex formation. It is based on the use of the collective variable ansatz which is 
constructed by taking into account the translational and internal degrees of freedom of a soliton as well as interactions between solitons and solitons with radiation:

$$
u(x, t)=u^{(\mathrm{s})}(x, t ; l, X, R)+u^{(\mathrm{r})}(x, t) .
$$

Here $u^{(\mathrm{s})}$ is a solitonic part and $u^{(\mathrm{r})}(x, t)$ is a part of the solution corresponding to radiation. It turns out that the condition of the complex formation of the closely sited solitons can be found neglecting the radiation [10]. So the complex dynamics can be considered in the framework of the soliton ansatz:

$$
u^{(s)}=4 \arctan \left\{\exp \left(\frac{x-X}{l}-R\right)\right\}+4 \arctan \left\{\exp \left(\frac{x-X}{l}+R\right)\right\},
$$

which is prompted by the forms of the wobbler equation (13) and the exact solution in equation (16). Here $l=l(t), X=X(t)$ and $R=R(t)$ are functions of time. Functions $l(t)$ and $X(t)$ describe the changing of the effective width of solitons and their translational motion, respectively. The function $R=R(t)$ corresponds to the changing separation between solitons, which is defined obviously as $L=2 l R$.

Inserting the ansatz into equation (11) and equation (12) we find the effective Lagrangian and Hamiltonian of two interacting solitons in strongly dispersive media. Details of the calculation of explicit expressions for the kinetic and potential energies can be found in [15]. Analysis of the potential energy of two interacting solitons as function of parameters $l$ and $R$ for $\beta_{0}=3 / 4$ exhibits a principal result: in the dispesive medium the repulsion between identical topological solitons gives place to the attraction at the point of parameters of exact soliton-complex solution.

Formation of the "excited" soliton-complex states is provided by another mechanism based on the soliton-radiation interaction. This leads to the radiation locking and results in some kind of the interference effect. To demonstrate this fact we investigated the "excited" states and their dynamic characteristics numerically in the dispersive double sine-Gordon equation and studied the effect of a magnetic field on the soliton binding process [15]. We found a full agreement in pictures of the formation of soliton-complex states in the dDSGE and piecewise model.

Thus the formation of soliton complexes turns out to be the universal effect in nonlinear strongly-dispersive media. This phenomenon is of both theoretical and practical interest. From the theoretical point of view it is very interesting that the dispersion produces a discrete spectrum for a nonlinear eigenvalue problem for purely solitonic solutions. The present investigation shows that the higher dispersive terms in the nonlinear wave equations effect much more essentially than the small perturbations. The dispersion causes the strong dissipation of energy of the moving $2 \pi$-soliton, but also makes it possible to create the bound soliton complexes consisting of two or more $2 \pi$-solitons which can move radiationlessly.

From the experimental point of view the soliton complexes may be very attractive for the use in energy and information transfer processes. In particular, the discrete arrays of Josephson junctions are usually described by one-dimensional dispersive sine-Gordon models. The fluxons, the $2 \pi$-kinks, in these systems could form the soliton complexes with specific properties discussed above. Therefore, Josephson 
junction arrays may be considered as suitable candidates for real experiments, in which the soliton complexes would manifest themselves.

Other examples of appropriate physical systems are the low-dimensional ferroand antiferromagnets. The nonlinear dynamics of dislocations [1] is another potential field of application of the obtained results. Thus there are many experimental possibilities to observe the occurrence of stationary soliton-complex dynamics in dispersive media.

\section{References}

1. Peyrard M., Kruskal M.D. Kink dynamics in the highly discrete sine-Gordon system. // Physica D, 1984, vol. 14, p. 88-102.

2. Boesch R., Willis C.R., El-Batanouny M. Spontaneous emission of radiation from a discrete sine-Gordon kink. // Phys. Rev. B, 1989, vol. 40, No. 4, p. 2284-2296.

3. Bogdan M.M., Kosevich A.M., Voronov V.P. Spectra of linear excitations of solitons in one-dimensional models of lattice theory. In: Soliton and Applications (Makhankov V.G., Fedyanin V.K., Pashaev O.K., eds.), Singapore, World Scientific, 1989, p. 231-243.

4. Nakajima K., Onodera Y., Nakamura T., Sato R. Numerical analysis of vortex motion on Josephson structures. // J. Appl. Phys, 1974, vol. 45, p. 4095-4099.

5. Lonngren K. Experimental investigation of solitons in nonlinear dispersive transmission lines. In: Solitons in Action (K.Lonngren and A.C.Scott, eds.), New York, Academic Press, 1978, p. 138-162.

6. Alfimov G.L., Eleonskii V.M., Kulagin N.E., Mitskevich N.N. Dynamics of topological solitons in models with nonlocal interactions. // Chaos, 1993, vol. 3, No. 3, p. 405-414.

7. Kawahara T., Toh S. Pulse interactions in an unstable dissipative-dispersive nonlinear system. // Phys. Fluids, 1988, vol. 31, No. 8, p. 2103-2111.

8. Christov C.I., Maugin G.A., Velarde M.G. Well-posed Boussinesq paradigm with purely spatial higher-order derivatives. // Phys. Rev. E, 1996, vol. 54, No. 3, p. 36213638.

9. Bogdan M.M., Kosevich A.M. Radiationless motion of one-dimensional solitons in dispersive medium. In: Nonlinear Coherent Structures in Physics and Biology. (Spatschek K.H., Mertens F.G., eds.), NATO ASI Series, Physics. vol. 329, New York, Plenum Press, 1994, p. 373-376.

10. Bogdan M.M., Kosevich A.M. Interaction of moving solitons in a dispersive medium and regimes of their radiationless motion. Proc. Estonian Acad. Sci. Phys. Math., 1997, vol. 46, No. 1/2, p. 14-23.

11. Savin A.V. Hypersonic regimes of topological soliton motion. // Sov. JETP, 1995, vol. 108, No. 9, p. 1105-1116 (in Russian).

12. Peyrard M., Piette B., Zakrzewski W.J. Soliton-like behaviour in a modified sineGordon model. // Physica D, 1993, vol. 64, p. 355-364.

13. Condat C.A., Guyer R.A., Miller M.D. Double sine-Gordon chain. // Phys. Rev. B, 1983, vol. 27, No. 1, p. 474-494.

14. Kosevich A.M., Kovalev A.S. The supersonic motion of a crowdion. The onedimensional model with nonlinear interaction between the nearest neighbours. // Solid State Commun., 1973, vol. 12, No. 8, p. 763-765. 
15. Bogdan M.M., Kosevich A.M., Maugin G.A. Soliton-complex dynamics in strongly dispersive medium. // Phys. Rev E, 1999 (in press).

\title{
Формування солітонних комплексів в дисперсійних системах
}

\author{
М.М.Богдан ${ }^{1}$, А.М.Косевич ${ }^{1}$, Ж.А.Можен ${ }^{2}$ \\ 1 Фізико-технічний інститут низьких температур ім. Б.І.Вєркіна НАН \\ України, 310164 Харків, просп. Леніна, 47 \\ 2 Університет П'єра і Марії Кюрі, 75252 Париж, Франція
}

Отримано 15 вересня 1998 р.

Формулюється концепція солітонних комплексів в нелінійному дисперсійному середовищі. Показано, що взаємодіючі тотожні топологічні солітони у такому середовищі здатні утворювати солітонні комплекси, що рухаються без випромінювання. Вважається, що це явище є універсальним і зустрічається у різних фізичних системах. Солітонні комплекси і їхні збуджені стани описуються аналітично і чисельно як розв'язки нелінійних дисперсійних рівнянь з четвертою $\mathrm{i}$ вищими просторовимі і змішаними похідними. Ретельно вивчаються дисперсійні системи синус-Гордон, подвійний і потрійний синусГордон, а також кусково-лінійна модель. Досліджуються механізми і умови формування солітонних комплексів і особливості їхньої стаціонарної динаміки. Запропоновано феноменологічний підхід до описання комплексів і класифікацію усіх можливих їхніх станів. Стисло розглянуті кілька прикладів фізичних систем, в яких це явище може експериментально спостерігатись.

Ключові слова: солітонний комплекс, дисперсійне середовище, безвипромінювальний рух

PACS: $47.35 .+i, 02.70 .-c, 47.20 . K y, 62.30 .+d, 03.40 . K f$ 
